\title{
Improving patient safety in cardiothoracic surgery: an audit of surgical handover in a tertiary center
}

\author{
Natasha Johan Bauer \\ Department of Cardiothoracic \\ Surgery, Royal Brompton Hospital, \\ London, UK
}

This article was published in the following Dove Press journal:

Advances in Medical Education and Practice

27 May 2016

Number of times this article has been viewed

Background: Novel research has revealed that the relative risk of death increased by $10 \%$ and $15 \%$ for admissions on a Saturday and Sunday, respectively. With an imminent threat of 7-day services in the National Health Service, including weekend operating lists, handover plays a pivotal role in ensuring patient safety is paramount. This audit evaluated the quality, efficiency, and safety of surgical handover of pre- and postoperative cardiothoracic patients in a tertiary center against guidance on Safe Handover published by the Royal College of Surgeons of England and the British Medical Association.

Methods: A 16-item questionnaire prospectively audited the nature, time and duration of handover, patient details, operative history and current clinical status, interruptions during handover, and difficulties cross-covering specialties over a month.

Results: Just over half (52\%) of the time, no handover took place. The majority of handovers $(64 \%)$ occurred over the phone; two-thirds of these were uninterrupted. All handovers were less than 10 minutes in duration. About half of the time, the senior house officer had previously met the registrar involved in the handover, but the overwhelming majority felt it would facilitate the handover process if they had prior contact. Patient details handed over $100 \%$ of the time included name, ward, and current clinical diagnosis. A third of the time, the patient's age, responsible consultant, and recent operations or procedures were not handed over, potentially compromising future management due to delays and lack of relevant information. Perhaps the most revealing result was that the overall safety of handover was perceived to be five out of ten, with ten being very safe with no aspects felt to impact negatively on optimal patient care.

Conclusion: These findings were presented to the department, and a handover proforma was implemented. Recommendations included the need for a new face-to-face handover. A reaudit will evaluate the effects of these changes.

Keywords: audit, handover pro forma, cardiothoracic surgery

\section{Introduction}

The pressure on the National Health Service to provide 7-day services has been mounting steadily over the last few months with increasing media and political involvement. This has resulted in increasing scrutiny of weekend services and statistics, focusing on morbidity and mortality throughout the country. Novel research that looked at 14.8 million admissions in 2013-2014 revealed a 30-day mortality of 1.8\%, with a relative risk of death deemed to increase by $10 \%$ and $15 \%$ for admissions on a Saturday and Sunday, respectively. ${ }^{1}$ With the imminent threat of increasing working hours and shift-work patterns, a thorough and efficient handover has a pivotal role in ensuring patient safety is paramount.
Correspondence: Natasha Johan Bauer Department of Cardiothoracic Surgery, Royal Brompton Hospital, Sydney Street, London SW3 6NP, UK

Tel +447703804650

Email natashabauer@doctors.org.uk 


\section{Materials and methods}

Ethical approval was not required for this research at the Royal Brompton Hospital. The data analyzed was anonymous hence patient written consent was not relevant in this project. Evening handovers of pre- and postoperative cardiothoracic surgery patients between senior house officers (SHOs) and junior registrars were evaluated prospectively. A total of 36 handovers were audited over a period of 6 weeks. A 16-item questionnaire was designed based on guidelines published in two key documents by the British Medical Association ${ }^{2}$ and the Royal College of Surgeons of England. ${ }^{3}$ The quality and effectiveness of the handover was assessed using a mixture of dichotomous, open, and Likert scale questions, which identified incomplete transfer of information and communication gaps that had the potential to negatively impact patient safety. Criteria audited included nature, time and duration of handover, patient details, including operative history and current clinical status, interruptions during handover, difficulties when cross-covering a different specialty, and completion of the advanced life support course and advanced trauma and life support course. Most importantly, the SHOs were asked to rate the safety of the handover process on a Likert scale of $0-10$ ( 0 being very unsafe and 10 very safe) as well as what they thought would significantly improve the handover process to maximize patient safety.

\section{Results}

Just over half $(52 \%)$ of the time, no handover took place. The majority of handovers (64\%) occurred over the phone; twothirds of these were uninterrupted. All handovers were less than 10 minutes in duration. About half of the time, the SHO had previously met the registrar involved in the handover, but the overwhelming majority felt it would facilitate the handover process if they had prior contact. The duration of all handovers was less than 10 minutes. Patient details handed over $100 \%$ of the time included name, ward, and current clinical diagnosis. A third of the time, the patient's age, responsible consultant, and recent operations or procedures were not handed over, which could compromise decisions made for future managements due to delays and lack of relevant information. Perhaps the most revealing result was that the overall safety of handover was perceived to be five out of ten, with ten being very safe with no aspects felt to impact negatively on optimal patient care.

\section{Conclusion}

This study of postoperative handovers highlighted five key aspects that determine a successful handover: use of a standardized process (checklist or protocol), minimal interruptions, focused patient handover (only clinical information), clinicians being present during handover, and education. In this subset of patients, it is crucial that early signs of clinical deterioration are recognized, managed, and escalated to senior members of the team. A cardiothoracic handover protocol has been developed to facilitate this process, and junior members of the team have been involved in a teaching and training session specifically addressing communication and thoroughness of information.

\section{Disclosure}

The author reports no conflicts of interest in this work.

\section{References}

1. Freemantle N, Ray D, McNulty D, et al. Increased mortality associated with weekend hospital admission: a case for expanded seven day services? BMJ. 2015;351:h4596.

2. The Royal College of Surgeons of England. Safe Handover: Guidance from the Working Time Directive Working Party. London, UK: The Royal College of Surgeons of England (RCS); 2007.

3. British Medical Association. Safe Handover: Safe Patients. London, UK: BMA; 2004.
Advances in Medical Education and Practice

\section{Publish your work in this journal}

Advances in Medical Education and Practice is an international, peerreviewed, open access journal that aims to present and publish research on Medical Education covering medical, dental, nursing and allied health care professional education. The journal covers undergraduate education, postgraduate training and continuing medical education

\section{Dovepress}

including emerging trends and innovative models linking education, research, and health care services. The manuscript management system is completely online and includes a very quick and fair peer-review system. Visit http://www.dovepress.com/testimonials.php to read real quotes from published authors. 